\title{
Combined Surface-Enhanced Raman Scattering Emissions for High-Throughput Optical Labels on Micrometer-Scale Objects
}

\author{
Meng-Yue Gao ${ }^{\dagger}$, Qiao Chen ${ }^{\dagger}$, Wei $\mathrm{Li}^{\ddagger}$, Ai-Guo Shen ${ }^{\dagger *}$, Ji-Ming $\mathrm{Hu}^{\dagger *}$ \\ ${ }^{\dagger}$ Key Laboratory of Analytical Chemistry for Biology and Medicine (Ministry of Education), College of \\ Chemistry and Molecular Sciences, Wuhan University, Wuhan 430072, People's Republic of China \\ tHubei Key Laboratory of Biomass Fiber and Eco-dyeing \& Finishing, College of Chemistry and Chemical \\ Engineering, Wuhan Textile University, Wuhan 430073, People’s Republic of China
}

\section{Corresponding Author}

agshen@whu.edu.cn

jmhu@whu.edu.cn

\section{Content}

TEM images of the as-prepared $\mathrm{Au} @ \mathrm{PBA}(\mathrm{Cu}) \mathrm{NPs}$ etched by $\mathrm{K}_{3}\left[\mathrm{Fe}(\mathrm{CN})_{6}\right]$ for different time .....S-2

The influence of etching time on the size, UV spectra and Raman intensity.

TEM images of Au@PBA(Cu) core-shell NPs prepared by adding the same amounts of precursors at different rate.

TEM images of the as-prepared Au@PBA(Cu) NPs prepared by adding different amounts of precursor reactants at the fixed concentration.

TEM images of Au@PBA core-shell NPs with various shell thicknesses.

TEM images of Au@PBA core-shell NPs.

TEM images of the as-prepared Au nanostars.

TEM images of the as-prepared Au nanostars @ PBA $(\mathrm{Cu})$ NPs and Au nanorods @PBA(Cu) NPs.

Power X-ray diffraction (XRD) patterns of Au@PBA core-shell NPs.

Electron microprobe of the Au@PBA NPs.

The simulated Raman spectra of the Au@PBA NPs.

The stablity of the Au@PBA NPs.

Particle sizes of Au NPs, Au@CN NPs, Au@PBA NPs, Au@PBA@pLL NPs, and Au@PBA@pLL@Apt NPs.

The TEM images of bacteria incubated with c-SERS tags and the corresponding SERS spectra.S-13

SERS imaging and SERS spectra of a model bacteria incubated with c-SERS tags for $3 \mathrm{~h}$.

SERS imaging and SERS spectra of four kinds of bacteria incubated with c-SERS tags for $3 \mathrm{~h}$. . S-18 
TEM images of the as-prepared Au@PBA $(\mathrm{Cu})$ NPs etched by $\mathrm{K}_{3}\left[\mathrm{Fe}(\mathrm{CN})_{6}\right]$ for different time

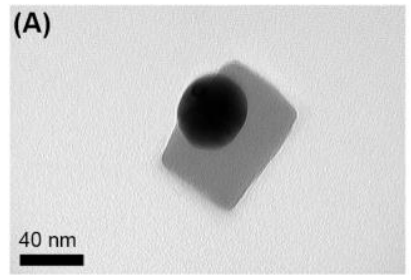

(B)

(C)

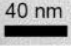

(D)
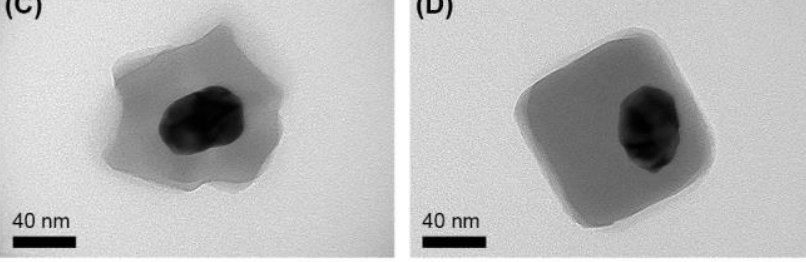

(E)

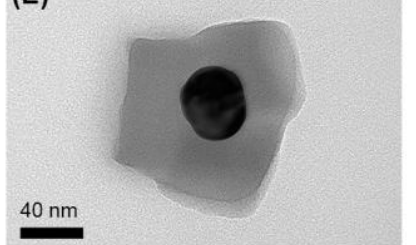

Figure S1. TEM images of the as-prepared $\mathrm{Au} @ \mathrm{PBA}(\mathrm{Cu}) \mathrm{NPs}$ etched by $\mathrm{K}_{3}\left[\mathrm{Fe}(\mathrm{CN})_{6}\right](0.5 \mathrm{mM})$ for $(\mathrm{A}) 5 \mathrm{~min}$, (B) $10 \mathrm{~min}$, (C) $30 \mathrm{~min}$, (D) $60 \mathrm{~min}$, and (E) $180 \mathrm{~min}$. 
The influence of etching time on the size, UV spectra and Raman intensity.
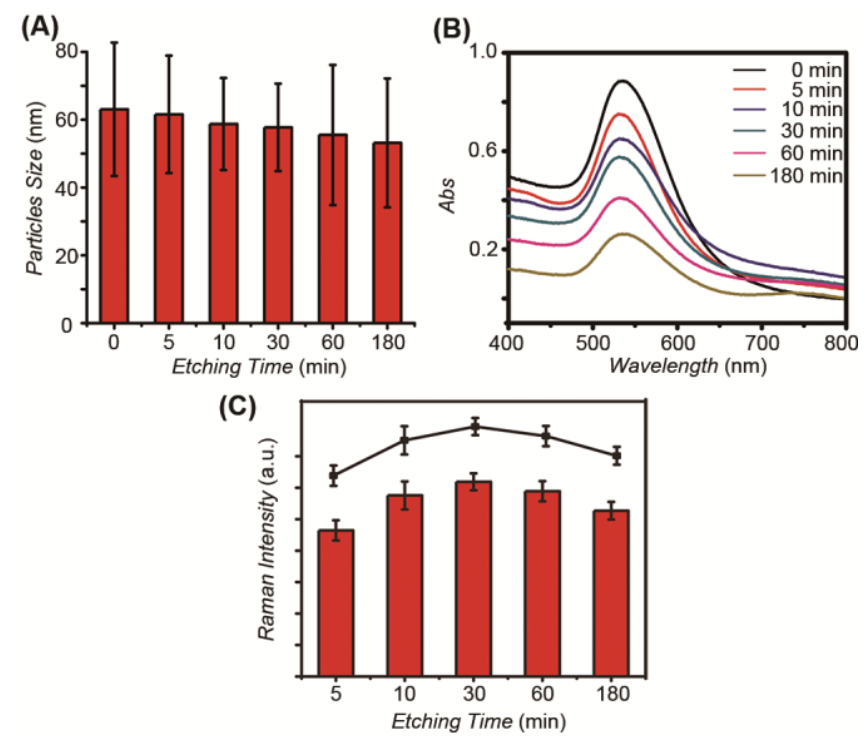

Figure S2. (A) Particle size of Au NPs etched by $\mathrm{K}_{3}\left[\mathrm{Fe}(\mathrm{CN})_{6}\right]$ for different time. (B) UV-vis spectra of the asprepared $\mathrm{Au}$ NPs etched by $\mathrm{K}_{3}\left[\mathrm{Fe}(\mathrm{CN})_{6}\right](0.5 \mathrm{mM})$ for $0 \mathrm{~min}, 5 \mathrm{~min}, 10 \mathrm{~min}, 30 \mathrm{~min}, 60 \mathrm{~min}$, and $180 \mathrm{~min}$. With etching, the maximal absorption bands blue-shifted gradually, indicating the slight decrease of particle size. (C) Raman intensity at $2187 \mathrm{~cm}^{-1}$ for Au@PBA $(\mathrm{Cu})$ NPs prepared by treating the as-prepared Au NPs with $\mathrm{K}_{3}\left[\mathrm{Fe}(\mathrm{CN})_{6}\right](0.5 \mathrm{mM})$ for different time. All samples were measured with $633 \mathrm{~nm}$ laser excitation. The error bars represent the standard deviations of ten repeating experiments.

TEM images of Au@PBA(Cu) core-shell NPs prepared by adding the same amounts of precursors at different rate.
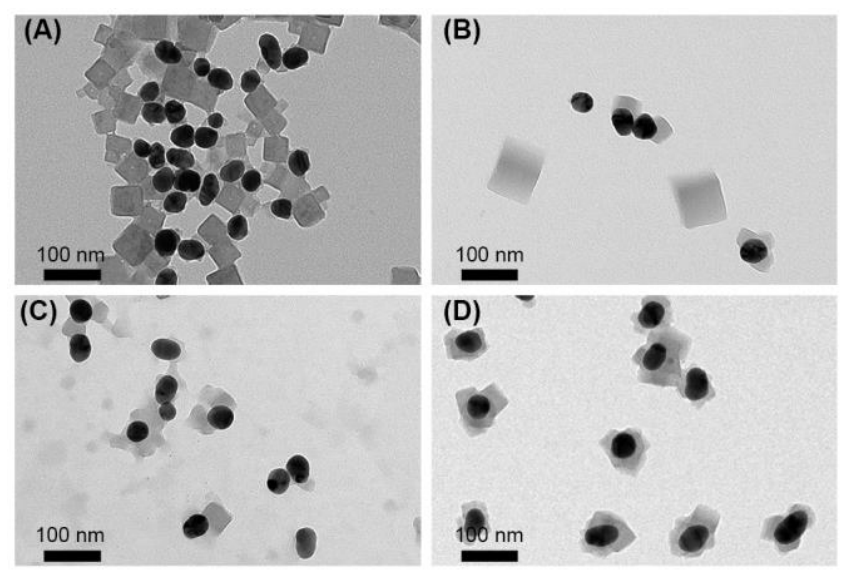

Figure S3. TEM images of $\mathrm{Au} @ \mathrm{PBA}(\mathrm{Cu})$ core-shell NPs prepared by adding the same amounts of precursors at different rate: (A) $2 \mathrm{~mL} / \mathrm{min}$, (B) $10 \mathrm{~mL} / \mathrm{h}$, (C) $5 \mathrm{~mL} / \mathrm{h}$, and (D) $2 \mathrm{~mL} / \mathrm{h}$. 
TEM images of the as-prepared Au@PBA(Cu) NPs prepared by adding different amounts of precursor reactants at the fixed concentration.
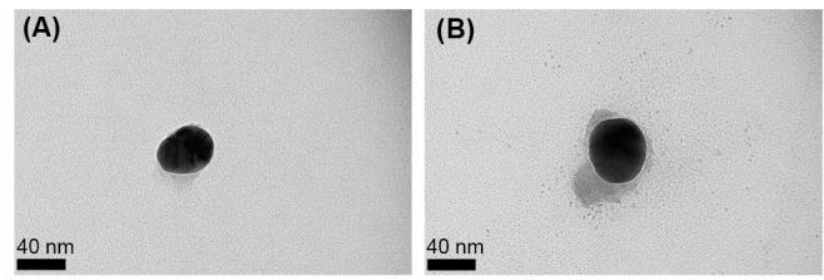

(C)
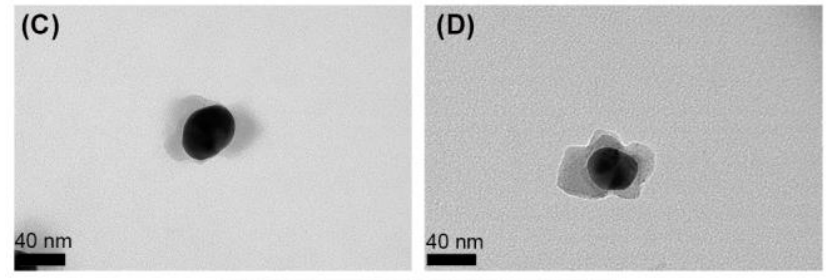

(E)

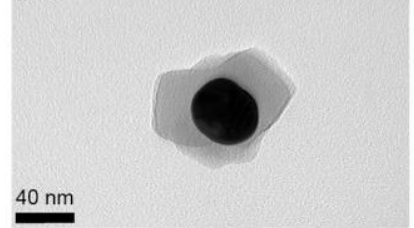

Figure S4. TEM images of the as-prepared Au@PBA(Cu) NPs prepared by adding different amounts of precursor reactants at the fixed concentration (0.1 mM): (A) $200 \mu \mathrm{L}$, (B) $500 \mu \mathrm{L}$, (C) $1000 \mu \mathrm{L}$, (D) $1500 \mu \mathrm{L}$, and (E) $2000 \mu \mathrm{L}$. 
TEM images of Au@PBA core-shell NPs with various shell thicknesses.
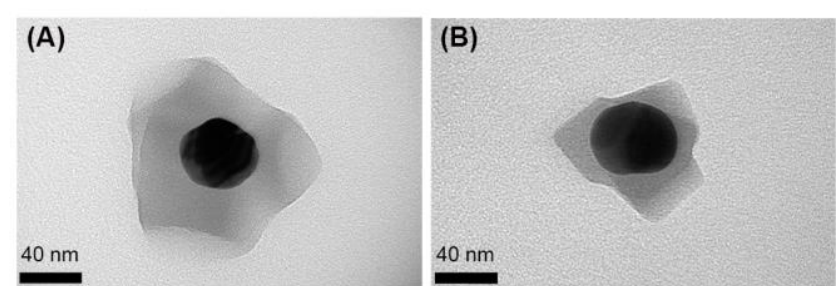

(C)

(D)
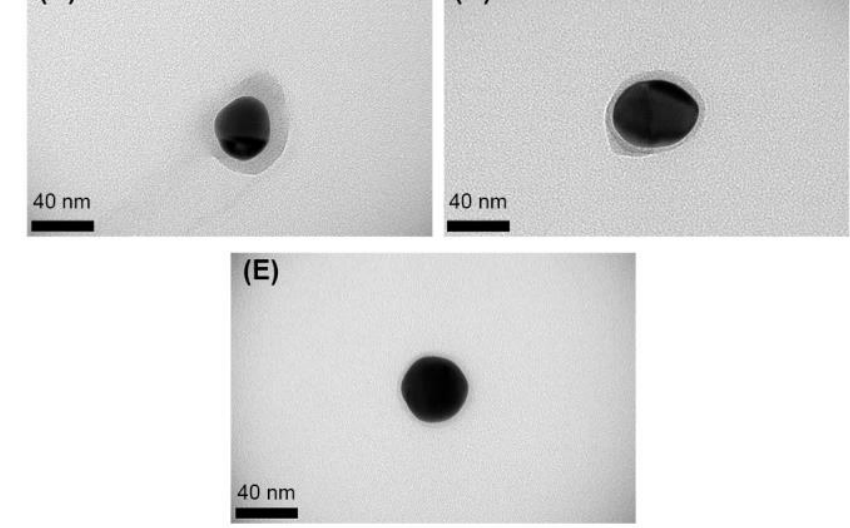

Figure S5. TEM images of Au@PBA core-shell NPs with various shell thicknesses. The thickness of PBA shell increased with the addition of PBA precursors: (A) $1000 \mu \mathrm{M}$, (B) $100 \mu \mathrm{M}$, (C) $50 \mu \mathrm{M}$, and (D) $10 \mu \mathrm{M}$, (E) $1 \mu \mathrm{M}$. The error bars represent the standard deviations of ten repeating experiments. 
TEM images of Au@PBA core-shell NPs.

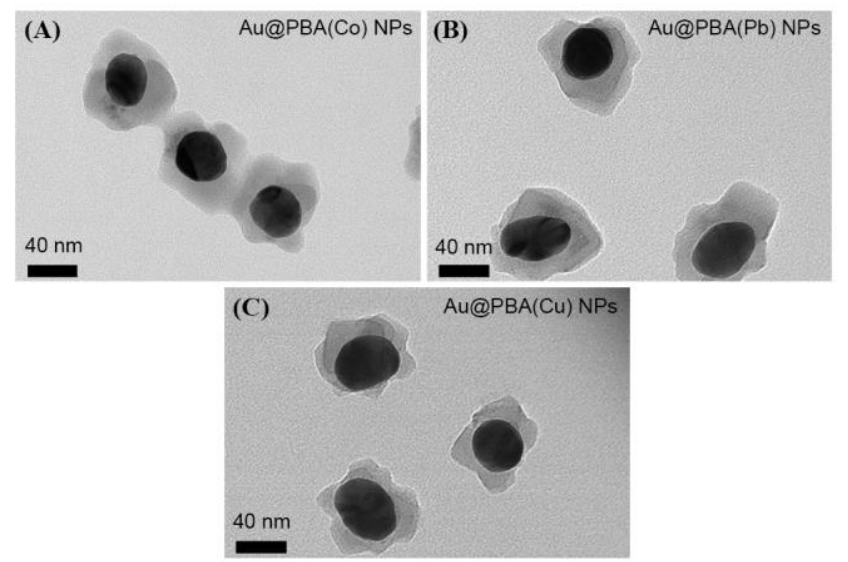

Figure S6. TEM images of (A) the Au@PBA(Co) NPs, (B) Au@PBA(Pb) NPs, and (C) Au@PBA(Cu) NPs.

TEM images of the as-prepared Au nanostars.

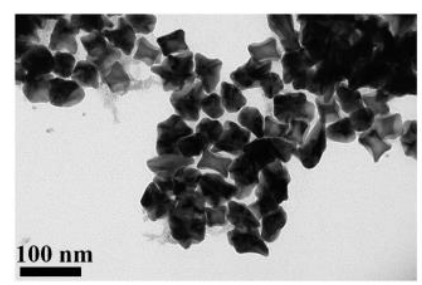

Figure S7. TEM images of the as-prepared Au nanostars

TEM images of the as-prepared Au nanostars @PBA(Cu) NPs and Au nanorods @PBA(Cu) NPs.
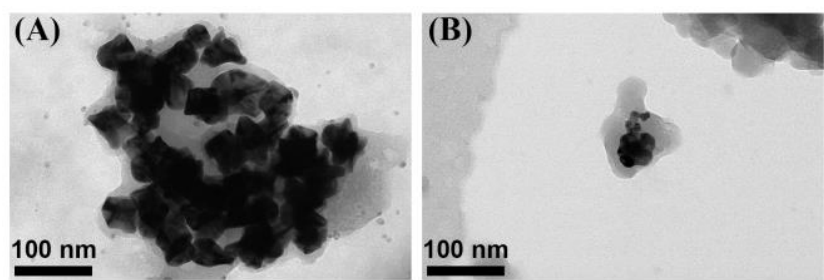

(C)

(D)
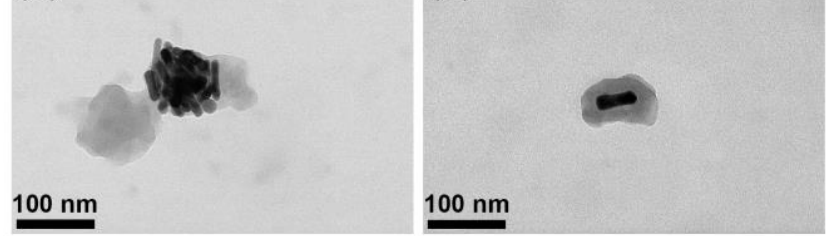

Figure S8. TEM images of the as-prepared Au nanostars @ PBA $(\mathrm{Cu}) \mathrm{NPs}$ and Au nanorods @PBA(Cu) NPs. 
Power X-ray diffraction (XRD) patterns of Au@PBA core-shell NPs.
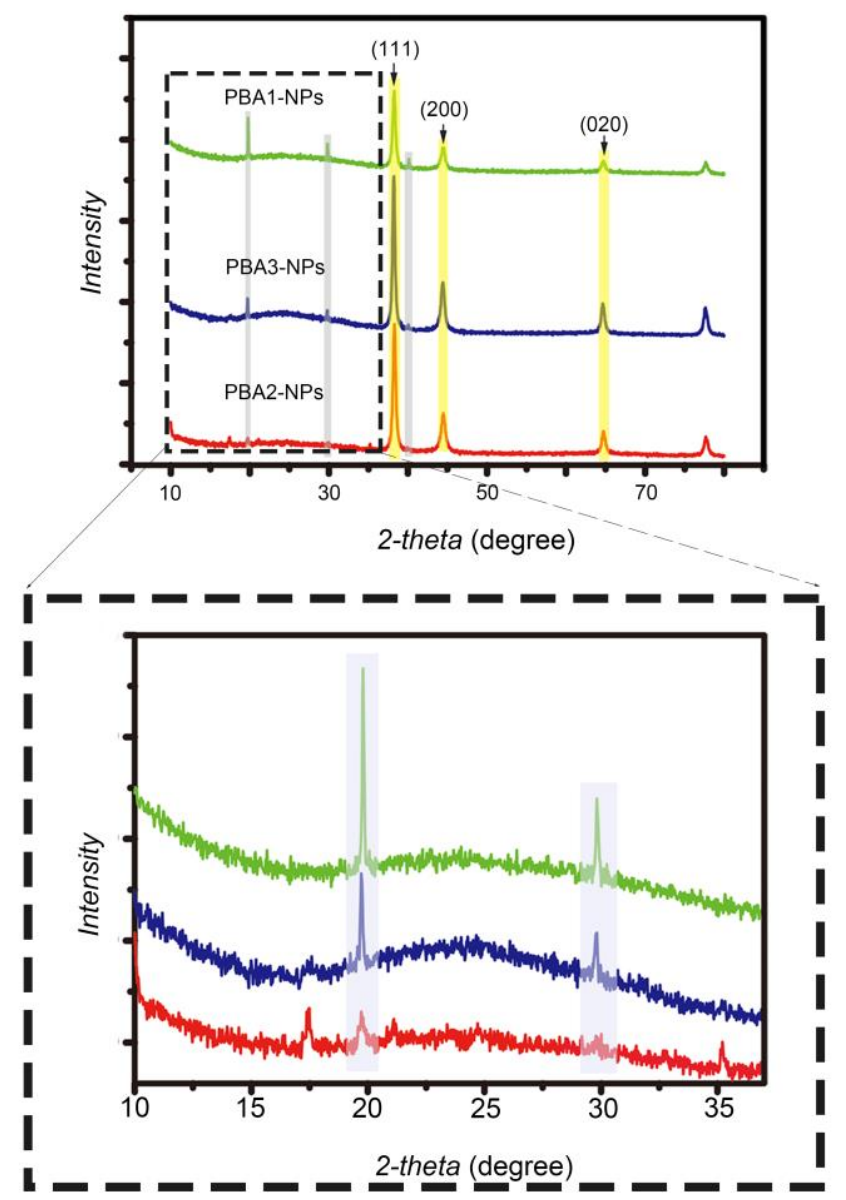

Figure S9. Power X-ray diffraction (XRD) patterns of Au@PBA(Pb) NPs (PBA1, green), Au@PBA(Co) NPs (PBA2, red), and Au@PBA(Cu) NPs (PBA3, blue). 
Electron microprobe of the Au@PBA NPs.
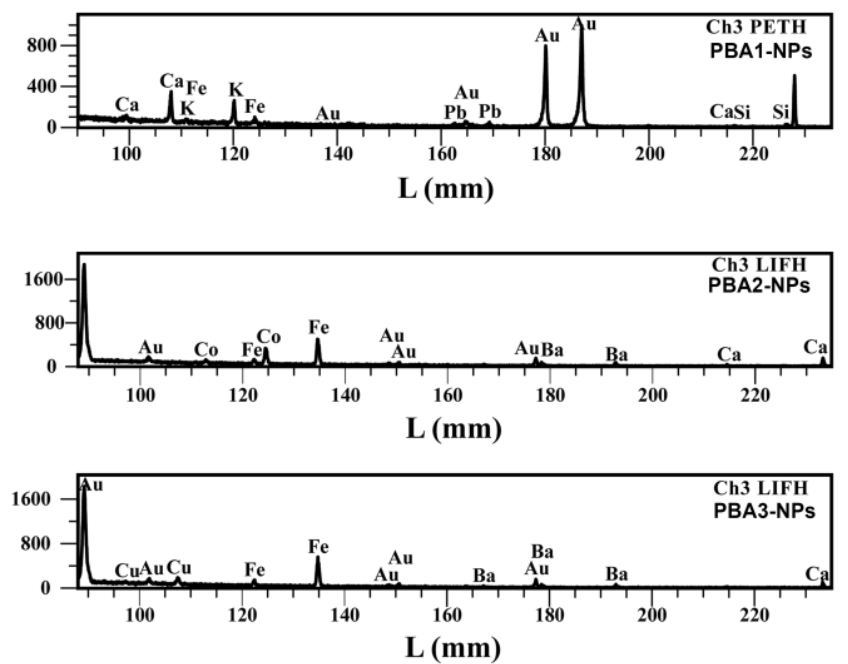

Figure S10. Electron microprobe of the Au@PBA NPs. PBA1-NPs, PBA2-NPs and PBA3-NPs respectively represent $\mathrm{Au} @ \mathrm{PBA}(\mathrm{Pb}) \mathrm{NPs}, \mathrm{Au} @ \mathrm{PBA}(\mathrm{Co}) \mathrm{NPs}$ and $\mathrm{Au} @ \mathrm{PBA}(\mathrm{Cu}) \mathrm{NPs}$. 
The simulated Raman spectra of the Au@PBA NPs.

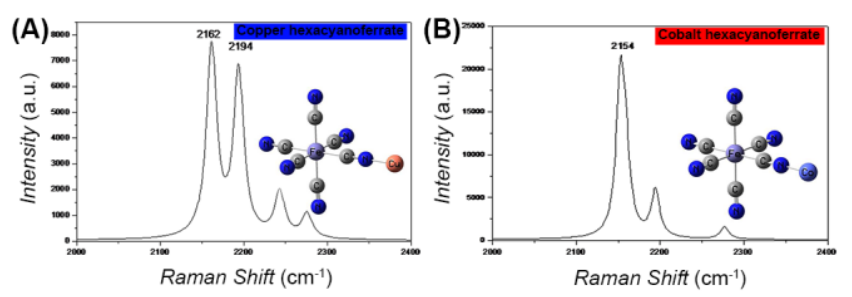

Figure S11. The simulated Raman spectra of (A) Au@PBA(Cu) NPs and (B) Au@PBA(Co) NPs acquired by theoretical calculations. 
The stablity of the Au@PBA NPs.

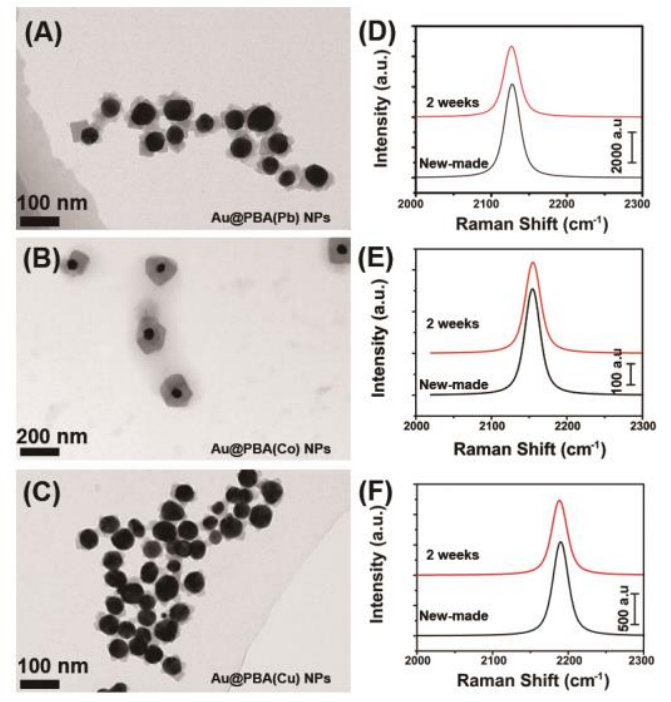

Figure S12. (A) (C) the TEM images of Au@PBA NPs and SERS spectra of Au@PBA NPs laying for 2 weeks at room temperature. 
Dynamic light scattering (DLS) data for the Au NPs, Au@CN NPs, Au@PBA NPs, Au@PBA@pLL NPs, and Au@PBA@pLL@Apt NPs.
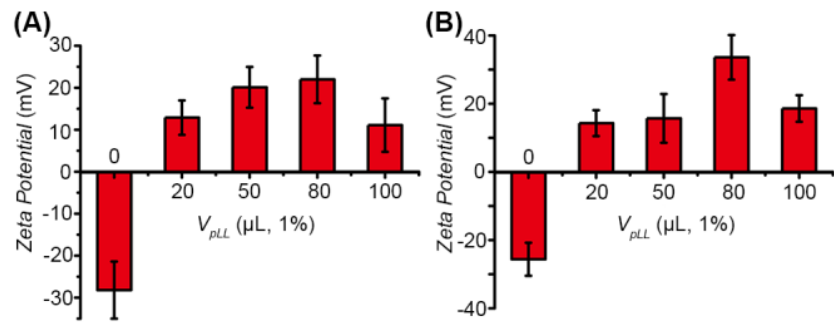

(C)

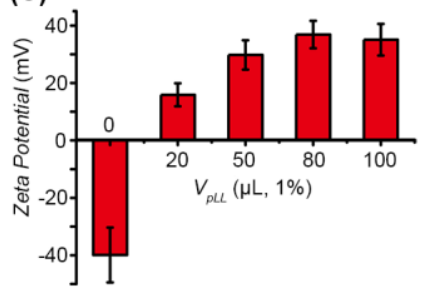

Figure S13. Dynamic light scattering (DLS) data for (A) Au@PBA(Pb)@Pll NPs, (B) Au@PBA(Co)@pLL NPs, and $(\mathrm{C})$ Au@PBA(Cu)@pLL NPs, which were prepared by adding different amounts of pLL aqueous solution $(10 \%, w): 0 \mu \mathrm{L}, 20 \mu \mathrm{L}, 50 \mu \mathrm{L}, 80 \mu \mathrm{L}$, and $100 \mu \mathrm{L}$. 
Particle sizes of Au NPs, Au@CN NPs, Au@PBA NPs, Au@PBA@pLL NPs, and Au@PBA@pLL@Apt NPs.
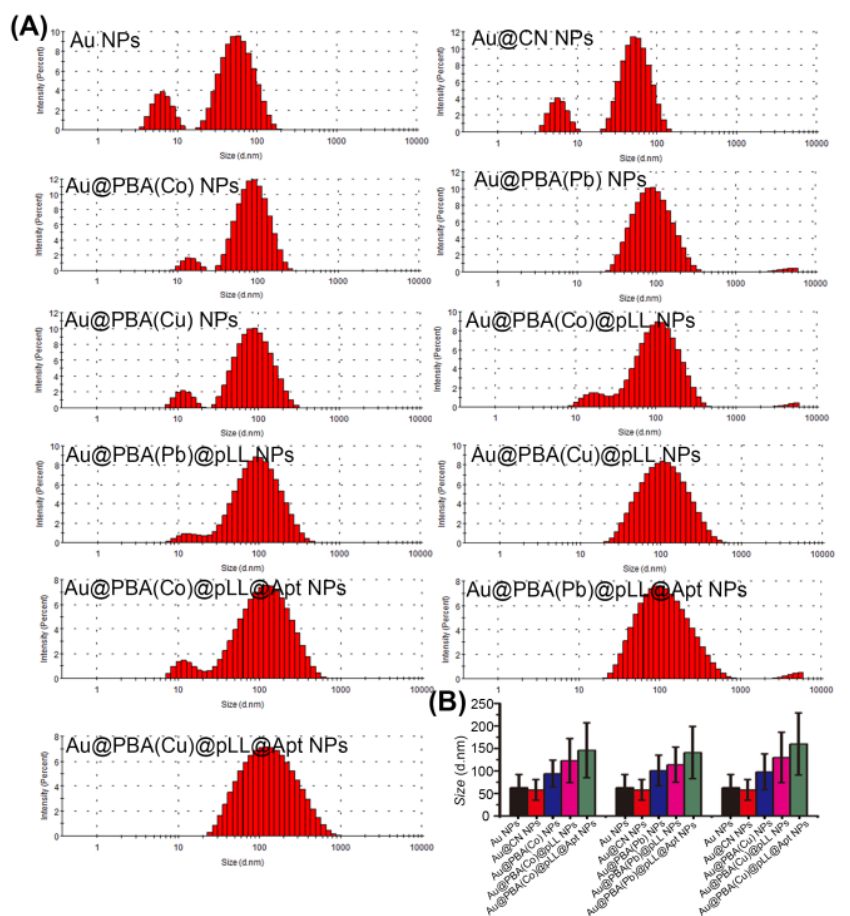

Figure S14. (A) Particle sizes of Au NPs, Au@CN NPs, Au@PBA NPs, Au@PBA@pLL NPs, and Au@PBA@pLL@Apt NPs. (B) Particle sizes of the above samples were measured by DLS. The error bars represent the standard deviations of three samples measured independently. 
The TEM images of bacteria incubated with c-SERS tags and the corresponding SERS spectra.
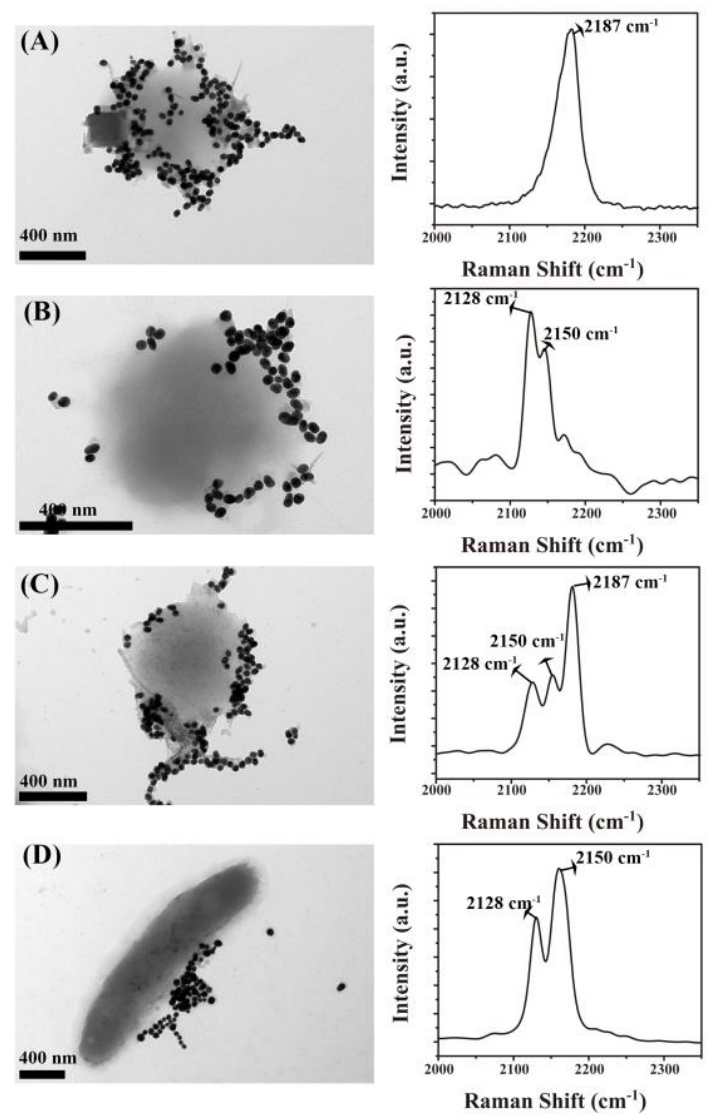

Figure S15. The TEM images of bacteria incubated with c-SERS tags and the corresponding SERS spectra: S. Aureus incubated with PBA2 (A); S. Aureus incubated with PBA1+PBA2 (B); S. Aureus incubated with PBA1+PBA2+PBA3 (C); E. Coli incubated with PBA1+PBA2 (D). 
SERS imaging and SERS spectra of a model bacteria incubated with c-SERS tags for 3h.

Bright Field

$2128 \mathrm{~cm}^{-1}$

$2150 \mathrm{~cm}^{-1}$

$2187 \mathrm{~cm}^{-1}$

Oerlapped

SERS Spectra
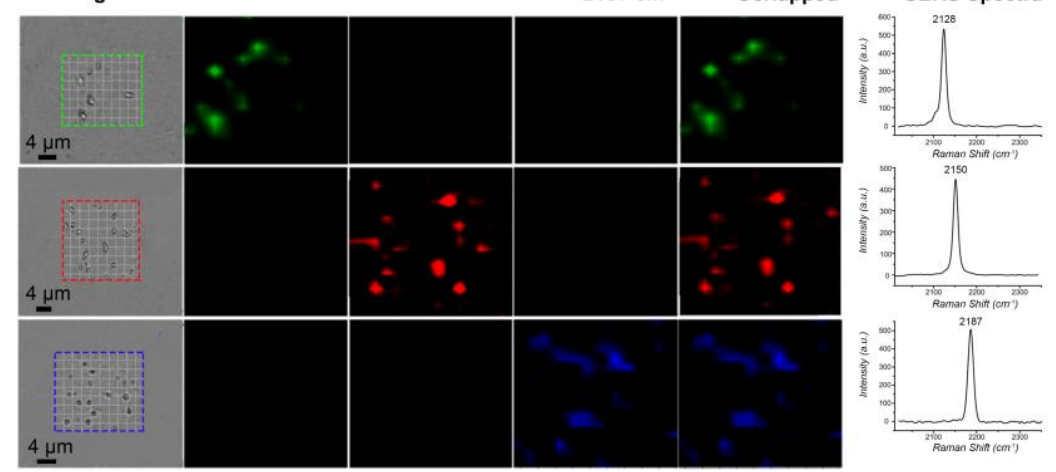

Figure S16. SERS imaging of a model bacteria incubated with c-SERS tags for $3 \mathrm{~h}$. For each sample incubated with PBA1, PBA2, or PBA3, imaging was drawn according to the intensity of three different Raman shifts respectively: 2128 (PBA1), 2150 (PBA2), and $2187 \mathrm{~cm}^{-1}$ (PBA3). 


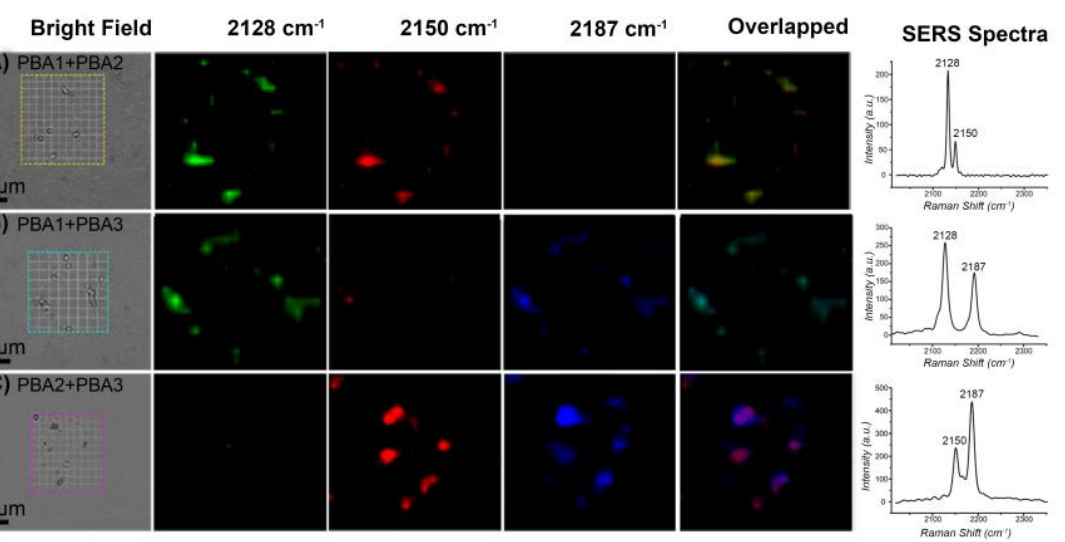

Figure S17. SERS imaging of a model bacteria incubated with c-SERS tags for $3 \mathrm{~h}$. For each sample incubated with $\mathrm{PBA} 1+\mathrm{PBA} 2$, PBA1+PBA3, or PBA2+PBA3, imaging was drawn according to the intensity of three different Raman shifts respectively: 2128 (PBA1), 2150 (PBA2), and $2187 \mathrm{~cm}^{-1}$ (PBA3). 


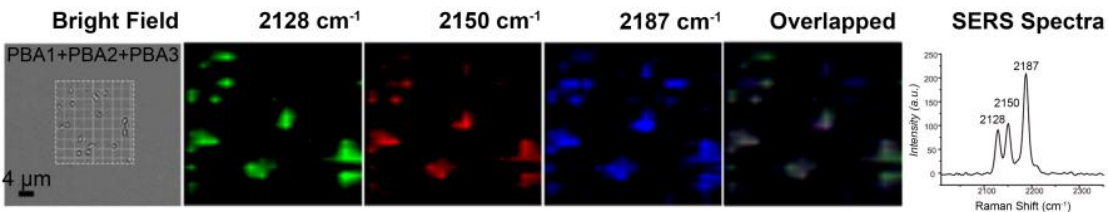

Figure S18. SERS imaging of a model bacteria incubated with c-SERS tags for $3 \mathrm{~h}$. For each sample incubated with $\mathrm{PBA} 1+\mathrm{PBA} 2+\mathrm{PBA} 3$, imaging was drawn according to the intensity of three different Raman shifts respectively: 2128 (PBA1), 2150 (PBA2), and $2187 \mathrm{~cm}^{-1}$ (PBA3). 


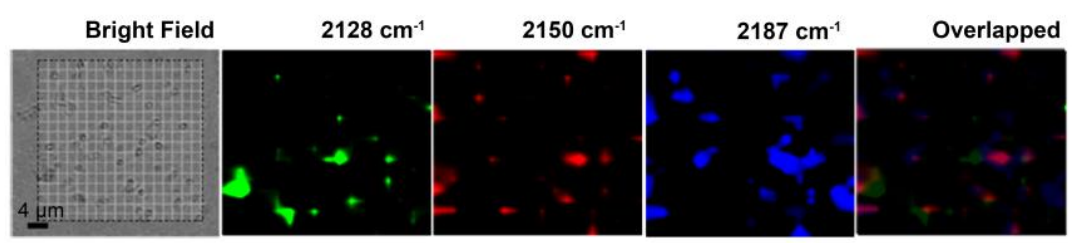

Figure S19. SERS imaging of a model bacteria incubated with c-SERS tags for $3 \mathrm{~h}$. For each sample incubated with PBA1, PBA2, PBA3, PBA1+PBA2, PBA1+PBA3, PBA2+PBA3, PBA1+PBA2+PBA3, imaging was drawn according to the intensity of three different Raman shifts respectively: 2128 (PBA1), 2150 (PBA2) and $2187 \mathrm{~cm}^{-1}$ (PBA3). (Scale bars: $\left.4 \mu \mathrm{m}\right)$ 
SERS imaging and SERS spectra of four kinds of bacteria incubated with c-SERS tags for $3 \mathrm{~h}$.

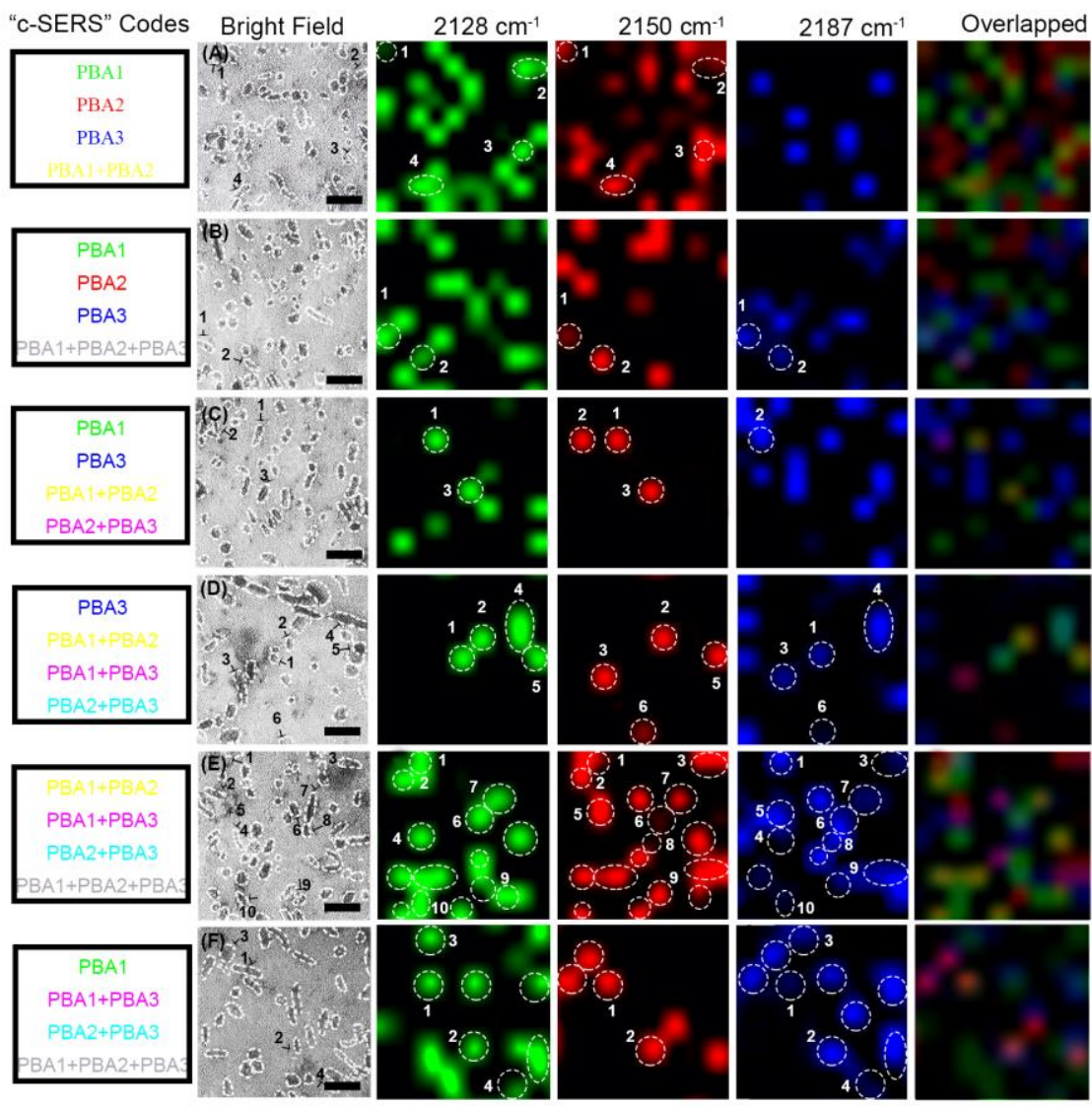

Figure S20. SERS imaging of the mixture of 4 common bacteria incubated with c-SERS tags for $3 \mathrm{~h}$, corresponding to figure 4. (Scale bars: $5 \mu \mathrm{m}$ ) (A) E. Coli, S. Aureus, P. Aeruginosa and B. Cereus were incubated with PBA1, PBA2, PBA3 and PBA1+PBA2 respectively; (B) E. Coli, S. Aureus, P. Aeruginosa and B. Cereus were incubated with PBA1, PBA2, PBA3 and PBA1+PBA2+PBA3 respectively; (C) S. Aureus, E. Coli, P. Aeruginosa and B. Cereus were incubated with PBA1, PBA3, PBA1+PBA2 and PBA2+PBA3 respectively; (D) E. Coli, S. Aureus, P. Aeruginosa and B. Cereus were incubated with PBA3, PBA1+PBA2, PBA1+PBA3 and PBA2+PBA3 respectively; (E) S. Aureus, E. Coli, P. Aeruginosa and B. Cereus were incubated with PBA1+PBA2, PBA1+PBA3, PBA2+PBA3 and PBA1+PBA2+PBA3 respectively; (F) S. Aureus, E. Coli, P. Aeru-ginosa and B. Cereus were incubated with PBA1, PBA1+PBA3, PBA2+PBA3 and PBA1+PBA2+PBA3 respectively. 

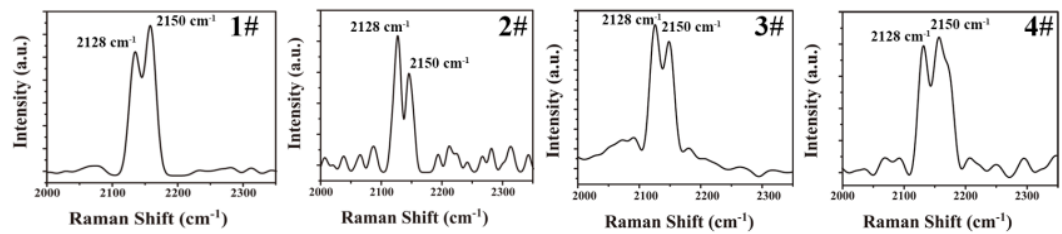

Figure S21. SERS spectra of the marked bacteria (1-4\#) in the figure S20 (A), incubated with c-SERS tags for $3 \mathrm{~h}$. (E. Coli, S. Aureus, P. Aeruginosa and B. Cereus were incubated with PBA1, PBA2, PBA3 and PBA1+PBA2 respectively.)

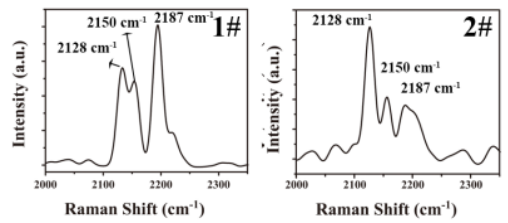

Figure S22. SERS spectra of the marked bacteria (1-2\#) in the figure S20 (B), incubated with c-SERS tags for 3h. (E. Coli, S. Aureus, P. Aeruginosa and B. Cereus were incubated with PBA1, PBA2, PBA3 and PBA1+PBA2+PBA3 respectively.)
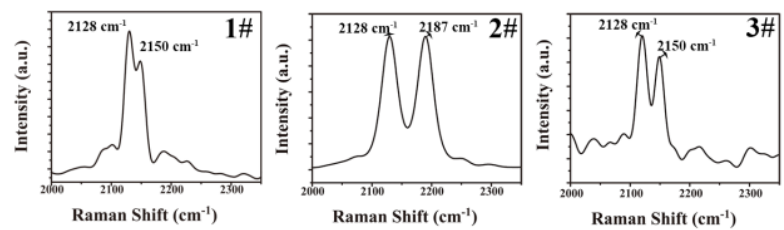

Figure S23. SERS spectra of the marked bacteria (1-3\#) in the figure S20 (C), incubated with c-SERS tags for 3h. (S. Aureus, E. Coli, P. Aeruginosa and B. Cereus were incubated with PBA1, PBA3, PBA1+PBA2 and PBA2+PBA3 respectively.).
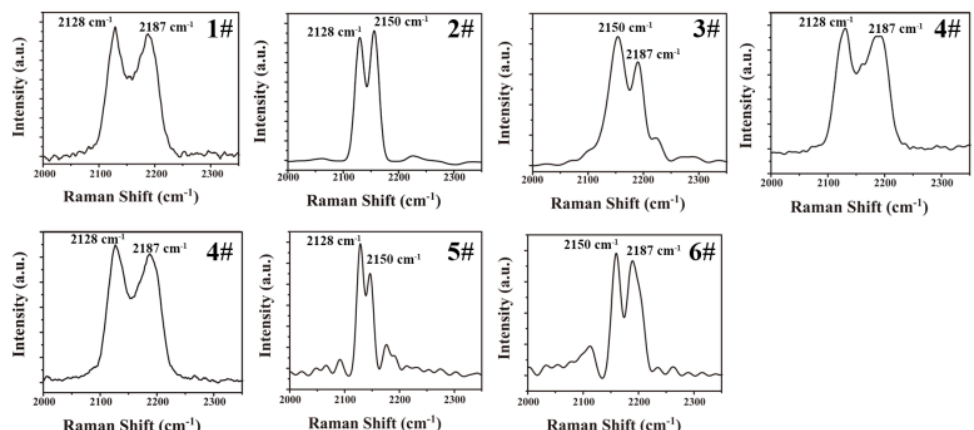

Figure S24. SERS spectra of the marked bacteria (1-6\#) in the figure S20 (D), incubated with c-SERS tags for $3 \mathrm{~h}$. (E. Coli, S. Aureus, P. Aeruginosa and B. Cereus were incubated with PBA3, PBA1+PBA2, PBA1+PBA3 and PBA2+PBA3 respectively.). 

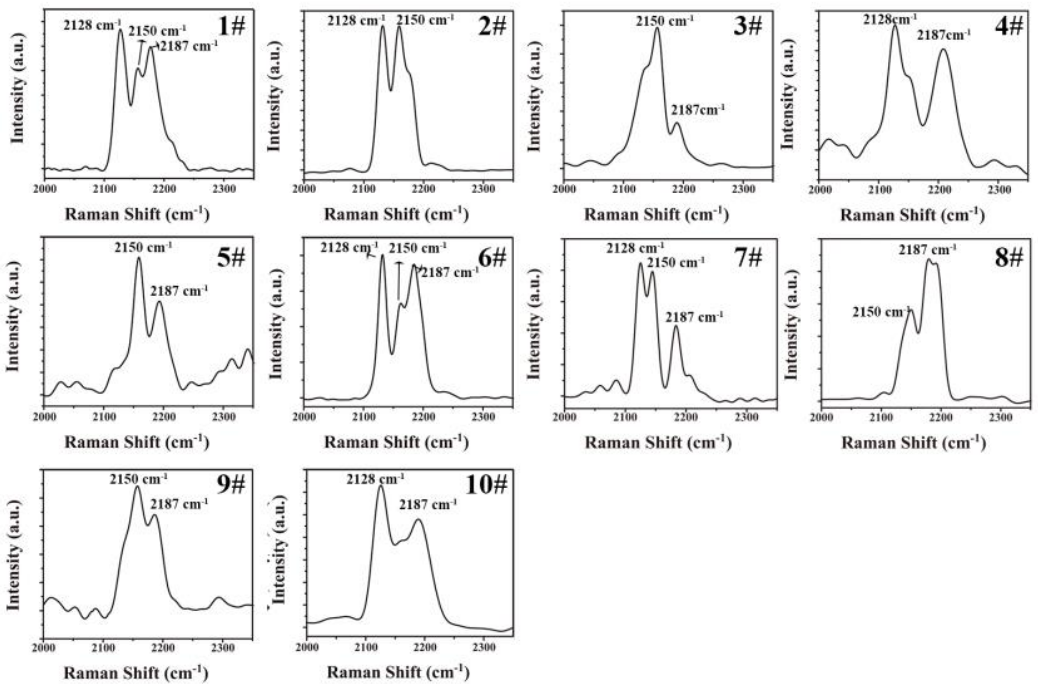

Raman Shift $\left(\mathrm{cm}^{-1}\right)$

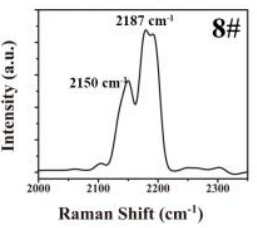

Figure S25. SERS spectra of the marked bacteria (1-10\#) in the figure S20 (E), incubated with c-SERS tags for 3h. (S. Aureus, E. Coli, P. Aeruginosa and B. Cereus were incubated with PBA1+PBA2, PBA1+PBA3, PBA2+PBA3 and PBA1+PBA2+PBA3 respectively.).
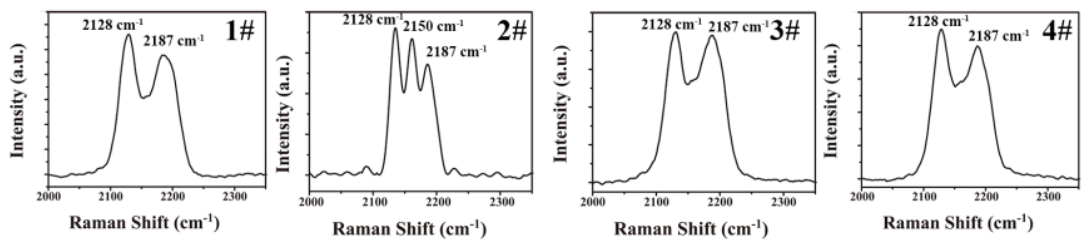

Figure S26. SERS spectra of the marked bacteria (1-4\#) in the figure S20 (F), incubated with c-SERS tags for $3 \mathrm{~h}$. (S. Aureus, E. Coli, P. Aeruginosa and B. Cereus were incubated with PBA1, PBA1+PBA3, PBA2+PBA3 and PBA1+PBA2+PBA3 respectively.). 